\title{
Performance Degradation Prediciton of Rolling Bearings based on BP Neural Networks
}

\author{
Xiaoxuan Qi ${ }^{1, a}$, Size Wang ${ }^{1, b}$,Jingfeng $\operatorname{Liu}^{2, c}$ \\ ${ }^{1}$ Shenyang University, Shenyang \\ ${ }^{2}$ Shenyang yidaxuntong Technology Co.,Itd \\ a13184088@qq.com, b641039302@qq.com, cliu_jingfeng@163.com
}

\begin{abstract}
Keywords: Evaluation of bearing performance degradationImage fusion; time-frequency analysis; BP neural network

Abstract. Roller bearings are commonly used components in rotating machinery and are pruned to be failure, which may cause the system break down and result in economic loss. Therefore, performance degradation prediction of rolling bearings is important to prevent any unexpected roller bearings failure. In this paper, time - frequency image fusion technology as well as the BP neural networks are utilized for fault feature extraction and prediction based on vibration signals.BP neural networks are used to learn the fault prediction features of vibration signals. Finally, the test data is used to testing the whole neural networks to establish the bearing condition monitoring model. Experimental results show that the proposed method achieves is about $80 \%$ accuracy to the bearing state recognition, and the recognition rate of the degraded performance stage is the highest, which can meet the engineering requirements.
\end{abstract}

\section{Introduction}

Rolling bearings are one of the most common parts in mechanical equipment which can withstand the load and has the function of relative function. Its performance can be fully experienced by a series of different degeneration until the complete failure. Its operating status directly affects the accuracy and reliability of mechanical equipment and its life span is one to two years. In recent years, many researchers have been working on data-driven predictive methods and analyzing the data of the life cycle of the bearing though some data processing methods ${ }^{[1]}$, and the feature quantity of the bearing degradation process is extracted and then analyzed and predicted by the prediction model. Among them, the prediction feature extraction and degradation state are identified as the basis of fault prediction, which is directly related to the accuracy of degradation state identification and the credibility of fault prediction ${ }^{[2]}$. The key point of this paper is to obtain effective prediction characteristics from the bearing life cycle data, and to ensure that these features have a good trend and consistency. As mentioned before, it also analyze the prediction characteristics through artificial neutral network and to achieve the evaluation and prediction of bearing's performance.

\section{Dataset}

The challenge data ${ }^{[3]}$ set includes run-to-failure vibration and temperature data collected from 17 bearings of the same model with Accelerated Life Tests (ALT). The vibration data for each case include two channels from two accelerometers placed radially on the external race of the bearing in vertical (channel 1) and horizontal (channel 2) directions respectively. The load is applied to the bearing radially in horizontal direction. The vibration data are collected every 10 seconds for a period of $1 / 10$ second at a sampling frequency of $25.6 \mathrm{kHz}$, which means 2560 samples per 10 second per channel. The total life of each case in the learning set and the censored life of that in the test set are listed in Table 1. 
Table 1 Total life/censored life of each bearing

\begin{tabular}{ccccc}
\hline Category & Index & Cond.1 & Cond.2 & Cond.3 \\
Learning set & 1 & 28020 & 9100 & 5140 \\
& 2 & 8700 & 7960 & 16360 \\
\hline \multirow{3}{*}{ Test set } & 3 & 18010 & 12010 & 3510 \\
& 4 & 11380 & 6110 & \\
& 5 & 23010 & 20010 & \\
& 6 & 23010 & 5710 & \\
\hline
\end{tabular}

\section{Performance evaluation based on BP network}

Preprocessing of the dataset Using MATLAB to extract data, respectively, The horizontal vibration signal I 2 and the vertical vibration signal I 2 of the data file are taken out, The amplitude of the horizontal vibration signal is analyzed.

Feature Extraction of Vibration Signals In order to obtain more fault information, thus fully and accurately express the bearing running state, So it is necessary to extract the characteristics of the time domain and time-frequency domain ${ }^{[4]}$ of the vibration signal.

\section{Feature extraction of vibration signal in Time domain}

(1) Maximum ${ }^{[5]} f_{1}$ :Collecting vibration signals in a certain period of time, And calculate the maximum value of the signal.(2) Mean $f_{2}$ :Collecting vibration signals in a certain period of time, And calculate the mean value of the signal. The signal $x(t)$ is discretely processed to obtain discrete digital

$$
\bar{X}=\frac{1}{n} \sum_{i=1}^{n}\left|x_{i}\right|
$$

(3) Peak $f_{3}$ : The maximum amplitude of the signal is in a certain period of $0.1 \mathrm{~s}$, which is called the peak. The expression of the peak is $x_{\max }=\max \left|x_{i}\right|$;(4) Root-mean-square $f_{4}$ : rams value is time averaged to reflect the energy of the signal. The signal is sampled after the discrete digital signal $x\left(t_{1}\right), x\left(t_{2}\right), x\left(t_{3}\right) \ldots x\left(t_{n}\right) ;(5)$ Kurtosis $f_{5}$ : The numerical statistics that reflect the distribution characteristics of random variables are normalized fourth order center matrices; (6) Waveform factor $f_{6}$ :

$$
K=\frac{x_{r m s}}{x^{\prime}}
$$

Where $K$ is the waveform index, $X_{r m s}$ is the mean square amplitude, and $X^{\prime}$ is the average mplitude; (7) Peak factor $f_{7}$ : To determine the abnormalities of rolling bearings, and can indicate whether the impact of the waveform indicators for the - peak factor; (8) Kurtosis factor $f_{8} ;$ (9) Pulse factor $f_{9} ;(10)$ Margin factor $f_{10}$.

\section{Feature extraction in time-frequency domain}

(1) the vibration signal of the vibration signal of time-frequency analysis USES the WVD time-frequency analysis, get the vibration signal of time-frequency images.

(2)co-occurrence matrix

As the texture is formed by the gray distribution in the spatial position of the recurrence, so there will be a certain degree of gray relations in the image space separated by a distance between the two 
pixels , that is, the image of the spatial correlation characteristics of gray. The gray-level co-occurrence matrix ${ }^{[6]}$ is a common method of describing texture by studying the spatial correlation of gray. $(a, b)$ values are selected by the characteristics of the texture period distribution. For smaller textures, small differences such as $(1,0),(1,10)$, and $(2,0)$ are selected. The covariant gray scale matrix is expressed as the pixel from the image gray scale $\mathrm{i}$, the cell position is $(\mathrm{x}, \mathrm{y})$, and the frequency is the same as the distance $\mathrm{d}$, the pixel of the gray scale $\mathrm{j}$, defined as

$$
\begin{aligned}
& P(i, j, D, \theta)=\left\{\left[(x, y),\left(x+D_{x}, y+D_{y}\right)\right]\right. \\
& \left.f(x, y)=i ; f\left(x+D_{x}, y+D_{y}\right)=j\right\} \\
& i, j=0,1, \mathrm{~L}, k-1
\end{aligned}
$$

where, $\theta$ is the generation direction of the co-occurrence matrix: $\left(0^{\circ}, 45^{\circ}, 90^{\circ}, 135^{\circ}\right)$.

(3) Time-frequency of characteristics

By the BARALDI experiment ${ }^{[7]}$, it is known that the two characteristic statistics based on energy, contrast and entropy are the most effective for classification and recognition.. Respectively calculate two kinds of characteristic value: energy $f_{11}$, entropy $f_{12}$, eigenvalues were taken in the direction of the four averages. Table 2 show the value of the first moment in different directions.

Table 2 eigenvalues at the first moment

\begin{tabular}{lrrrr}
\hline Feature & $0^{\circ}$ & $45^{\circ}$ & $90^{\circ}$ & $135^{\circ}$ \\
Energy & 0.332509, & 0.325793 & 0.333260 & 0.325242 \\
Entropy & 2.331535, & 2.482209, & 2.346601, & 2.486483 \\
\hline
\end{tabular}

Features analysis The characteristics of the horizontal vibration signal are analyzed in detail, as shown in Figure 3 for the horizontal vibration signal in the time domain of the 10 characteristics.
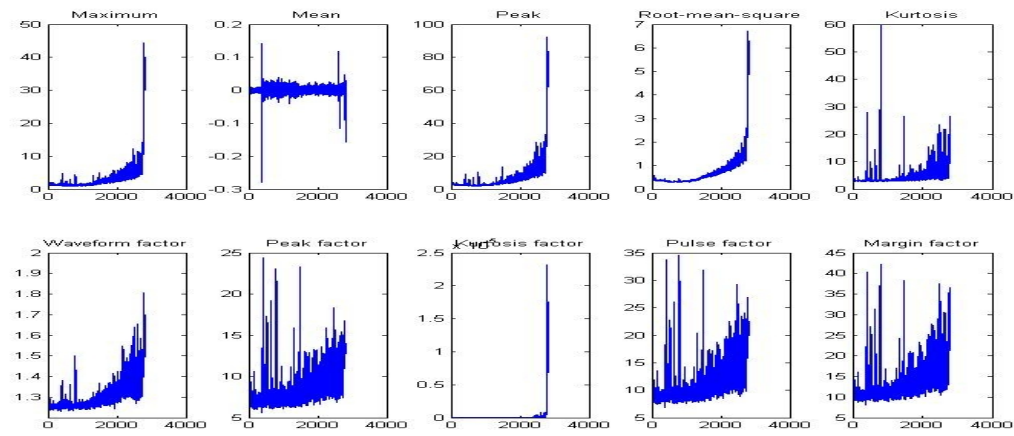

Figure 3 Time-frequency feature image

BP neural networks In the experiment, The training BP neural network training samples are the characteristics of the first group of Bearing1_1, the output tags are: the normal state, performance degradation status and failure status, second groups of test the samples: Bearing1_3, Bearing1_4, Bearing1_5, Bearing1_6, and Bearing1_7. The number of neural network is 3, The number of input neurons is 12 , the number of hidden layer neurons is 10 and the number of output layer neurons is about 3.

\section{Results and Analysis}

The whole life cycle data of two groups of the same type bearings are used for the experiment. The first group of data is used to determine the structure of the network, and the second groups of data are used to test the performance of the test. The test environment is set as follows: the speed of $1800 \mathrm{r} / \mathrm{min}$, the load is about $4000 \mathrm{~N}$, the sampling frequency of the acceleration sensor is set at $\mathrm{kHz}$, and the data acquisition time is $0.1 \mathrm{~s}$ ( 2560 data points) every $10 \mathrm{~s}$. Two groups of samples were collected with 2800 
and 870 samples respectively. The recognition rate of the network is about $90 \%$, and the recognition rate of the network is about $80 \%$, as shown in table three.

The performance prediction error is calculated as follows:

$$
E=\frac{T_{R}-T_{P}}{T_{R}} \times 100 \%
$$

For the built BP neural network input test samples, the results are shown in table 3.

Table 3 BP neural network prediction error

\begin{tabular}{cc}
\hline Bearing number & Prediction Error \\
Bearing1_3 & $100 \%-74.916 \%$ \\
Bearing1_4 & $100 \%-84.895 \%$ \\
Bearing1_5 & $100 \%-74.935 \%$ \\
Bearing1_6 & $100 \%-75.065 \%$ \\
Bearing1_7 & $100 \%-80.400 \%$ \\
\hline
\end{tabular}

\section{Conclusions}

In this paper, BP neural networks based rolling bearing condition recognition system is proposed. The bearing vibration signal is taken as the monitoring object, and the time domain and time-frequency domain features are extracted to form the feature vector of the model. The feature vector is input into the BP neural network for supervised feature learning, which reduces the feature dimension and determines the initial values of the network parameters ( $\mathrm{W}$ and $\mathrm{B}$ ). The prediction error of vibrations is analyzed. Experimental results show that the proposed algorithm has a high prediction accuracy, and has a good generalization ability for the recognition of the performance degradation of rolling bearings.

\section{Acknowledgements}

This work was financially supported by the Liaoning Natural Science Foundation (201602520), and Shenyang Program for Tackling Key Problems in Science and Technology(F15-126-9-00).

\section{Reference}

[1] FEMTO-ST, 'IEEE PHM 2012 DaTa Challenge," online website, last accessed on May 31, 2012. http://www.femto-st.fr/en/Research-departments/AS2M/Research-groups/PHM/IEEE-PHM-2012-D ata_challenge.php

[2] S. A. MchInerny and Y. Dai, "Basic vibration signal Processing for bearing fault detection,"

IEEE Transactions on Education, Vol 46(1), Feb 2003, pp. 149-156

[3] J. MacGregor and T. Kourti. "statistical process-control of multivariatre process," Control Engineering Practice, vol. March 1995, pp. 403-414.

[4] A. Sexena, J. Celaya, B. Saha, S. Saha, and K.Goebel, "Metrics for offine evaluation of prognostic performance," International Journal of Prognostics and Health Management, April 2010.

[5] N. Gebraeel, M. Lawley, R. Li, and J. Ryan, "Residual-life distributions from component degradation signals: A Bayesian approach," IIE Transactions, vol. 37, pp. 543-557, 2005.

[6] F. Cadini, E. Zio, and D. Avram, "Model-based Monte Carlo state estimation for condition-based component replacement," Reliability engineering and System Safety, vol. 94, pp. 752-758, 2009.

[7] Zhaokun Li, Xiaohui Zhao. BP artificial neural network based wave front correction for sensor-less free space optics communication[J]. Optics Communications, 2016. 